\title{
Situation sanitaire et nutritionnelle des ménages maliens en période de soudure
}

\author{
Medical and nutritional situation of the households Malians in period of welding
}

\author{
AG IKNANE A 1, DIARRA S 2, AG AYOYA M3, DJANGO DM 4, DIAWARA A 5, THIERRO TA6, NIANGALY A 7.
}

1: Maître assistant en Santé Publique à la FMPOS, chef service nutrition à l'INRSP Bamako

2. Médecin généraliste, Système d'alerte précoce (SAP) Bamako

3. Médecin nutritionniste, UNICEF, 73 Lodi Estate, New Dehli, 110-003, Inde

\section{Introduction :}

Au Mali, la situation alimentaire est demeurée inquiétante pendant plusieurs décennies. En 2005, 30 sites sentinelles avaient été établis pour surveiller la situation alimentaire et nutritionnelle des populations maliennes.

Matériel et méthodes :

Pour étendre l'expérience des sites sentinelles à tout le pays, une étude par échantillonnage stratifié a été réalisée en juin 2007 par le système d'alerte précoce (SAP). L'étude a concerné 5510 femmes en âge de procréer, 7293 enfants de moins de 5 ans repartis entre 3180 ménages installés dans 212 villages au niveau national.

Résultats :

La prévalence de l'héméralopie parmi les femmes a été estimée à $6 \%$. Celle de l'anémie clinique était 20\%. 50\% de femmes enceintes a bénéficié de la supplémentation de fer et $60 \%$ de femmes enceintes ont reçu un traitement prophylactique contre la malaria. $29 \%$ d'enfants âgés 0 à 59 mois sont tombé malades pendant les sept jours avant précédés l'étude (principalement diarrhée, IRA et paludisme présumé). L'allaitement exclusif est pratiqué dans $34 \%$ des cas.

L'émaciation affecte près de $10 \%$ d'enfants de moins de 5 ans. GAO est la région la plus affectée (16\%). Selon les systèmes de la vie, ce sont les systèmes de vie agromigration $(16 \%)$ qui ont enregistré les taux les plus élevés. Les taux les plus bas ont été observé dans le système de vie agro-coton - fruit avec $5 \%$.

Le retard de croissance affecte $25 \%$ d'enfants âgés 0 à 5 ans. La région de Sikasso est la plus affectée (33\%). Selon les systèmes de la vie, elle a atteint $41 \%$ d'enfants dans les systèmes de vie agro-fruit contre $14 \%$ dans les systèmes de vie salariat-commerce.

Il y a une corrélation forte entre le retard de croissance et l'insécurité alimentaire, la malnutrition et les maladies telles que la diarrhée, la rougeole, les IRA.

Conclusion et recommandation :

Les efforts du SAP doivent être soutenus par un meilleur suivi des interventions en matière de santé, alimentation et nutrition des populations.

Mots-clés : anémie, cécité, Emaciation, retard de croissance, diarrhée, Mali

Introduction:

In Mali, the nutritional situation remained worrying for several decades. In 2005, 30 sentinel sites tests had been established to monitor their food and nutrition situation.

Material and methods:

To extend the experience of sentinel sites throughout the country a study by stratified cluster sampling was
4. Maître assistant en anesthésie réanimation, CHU Gabriel Touré, Bamako

5. Maître assistant en Santé Publique, Directeur de l'ANEH, Bamako

6. Biologiste, gestionnaire des services de santé, INRSP, Bamako

7. Médecin de santé publique, Maître de recherche, INRSP, Bamako

conducted by Early Warning System in June 2007. The study involved 5510 women in age to procreate, 7293 children under 5 years, 3180 households and 212 villages at the national level.

Results: The prevalence of night blindness among the women is estimated at $6 \%$. That of clinical anaemia was $20 \%$. $50 \%$ of pregnant women practiced iron supplementation and $60 \%$ of pregnant women used a drug as a prophylactic against malaria. $29 \%$ of children aged 0 to 59 months fell ill during the seven days before the survey (mainly diarrhoea, ARI and suspected malaria). Exclusive breastfeeding has been found in $34 \%$ of children.

Wasting affects nearly $10 \%$ of children 0 to 5 years. GAO is the most affected region (16\%). According to the systems of life, are the strata agro-migration (16\%) has the highest rates. The lowest rates were observed in the strata agro-cotton -fruit (5\%).

Stunting affects $25 \%$ of children aged 0 to 5 years. The Sikasso region is the most affected (33\%). According to the systems of life, it reached $41 \%$ of children in agro-fruit strata against $14 \%$ in the strata wage-earning-trade.

There is a strong correlation between wasting and food insecurity, malnutrition and diseases such as diarrhoea, measles, ARI.

Conclusion and recommendation:

The efforts of the SAP must be supported for a better monitoring and improvement of the health and nutritional status of populations.

Keywords: anaemia, blindness, wasting, stunting, diarrhoea, Mali

\section{Introduction}

Le Mali, à l'image d'autres pays sahéliens, vit dans une situation d'insécurité alimentaire chronique faite de crises alimentaires conjoncturelles et récurrentes dues aux aléas climatiques. Selon le Système d'Alerte Précoce (SAP) « depuis près de 20 ans, les ménages maliens connaissent des difficultés alimentaires à une échelle plus ou moins grande une année sur trois ». Dans l'optique de suivre l'évolution de la situation alimentaire et nutritionnelle, 30 sites sentinelles tests avaient été mis en place, mais très rapidement le besoin d'une extension du système de suivi à l'ensemble du pays s'est vite fait ressentir. C'est dans ce cadre que le SAP/CSA, avec l'appui technique et financier de ses partenaires, a réalisé une "enquête de base sur la situation sanitaire et nutritionnelle des enfants de 0 à 58 mois et les femmes en âge de procréer pour l'extension des sites sentinelles" dont la première phase s'est déroulée en juin 2007. 


\section{MATERIELS ET METHODES.}

Une étude transversale stratifiée par sondage en grappe à deux degrés a été réalisée du 15 juin au 03 juillet 2007 auprès de 3180 ménages répartis dans 212 villages, 5494 femmes en âge de procréer dont 4626 mères d'enfants de 0 à 59 mois au nombre de 7259 dont 3803 garçons $(52 \%)$ et 3456 filles $(48 \%)$ repartis sur les 8 régions administratives du pays et le district de Bamako. L'ensemble du pays avait été subdivisé en douze (12) zones selon les systèmes de vie dominants combinant les variables socioéconomiques et géophysiques : agrocoton, agro-coton-fruit, agro-élevage, agro-migration ; agro-oignon, agro-pasteur, agro-rente, culture de décrue, pasteur, riz irrigué, riz submersion.salariat-commerce. Au premier degré, 212 villages ou quartiers, constituant les unités primaires de sondage, avaient été tirées de façon systématique avec une probabilité proportionnelle à la taille en ménages des villages.

Au second degré, un échantillon de 3180 ménages avait été sélectionné à partir de la liste des ménages, puis 15 ménages ont été tirés dans chaque village à probabilité égale. La taille de l'échantillon a été déterminée par la formule usuelle de Schwartz.

Des mesures anthropométriques (poids, taille, âge, sexe) de la population étudiée avaient été collecté. Les données anthropométriques avaient été normalisées à partir de la table standard OMS de décembre 2005 et un seuil < - 2 ET retenu pour définir la malnutrition chez l'enfant. La consommation de sel iodé a été appréciée à l'aide d'un test réalisé sur le sel consommé dans chaque famille au passage des enquêteurs avec le MBI KITS iode. L'allaitement exclusif avait été analysé à l'aide du croisement de la déclaration de la mère avec la consommation alimentaire des enfants selon le rappel des 24 heurs pour éliminer tout enfant ayant absorbé tout autre aliment que le lait maternel, l'eau plate y comprise [1]. La carence en fer et en vitamine $A$ avaient été appréciée à travers un examen clinique minutieux et un interrogatoire réalisé par des médecins.

Le test du $\mathrm{Chi}^{2}$ de Pearson avait été utilisé pour la comparaison des moyennes. Au plan éthique, un consentement préalable était obtenu et les interviews s'étaient déroulées dans le respect de l'intimité des ménages. Le logiciel SPSS.13.0 avait été utilisé pour l'épuration et l'analyse des données.

\section{Résultats}

La population des mères était majoritairement très jeune car 59\% d'entre elles avaient moins de 30 ans parmi lesquelles $14 \%$ avaient moins de 20 ans et $36 \%$ moins de 25 ans. La moyenne d'âge des femmes était de 28 ans, comparable dans toutes les régions excepté Gao dont l'âge moyen était de 31 ans.

La quasi-totalité des mères étaient mariées, soit $(94 \%)$ dans l'ensemble des régions. La proportion la plus importante de mères célibataires a été observée à Bamako avec (18\%), suivi de Kidal avec (9\%). II en était de même pour les femmes divorcées ou séparées avec $1.7 \%$ à Bamako et à $1,1 \%$ à Kidal.

Au niveau national, le taux d'analphabétisme était très élevé parmi les femmes en âge de procréer avec 75\% qui n'avaient reçu aucune éducation formelle. Seules
$10 \%$ des femmes en âge de procréer (FAP) avaient le niveau du premier cycle fondamental et $5 \%$ le second cycle du primaire. Pour le niveau secondaire il n'était que de $3 \%$ contre $0,3 \%$ pour le niveau supérieu. Par contre, près de $6 \%$ des FAP avaient reçu une éducation coranique. Au niveau régional, Koulikoro, Sikasso, Ségou et Mopti avaient enregistré les plus forts taux d'analphabétisme avec respectivement $91 \%, 92 \%, 84 \%$ et $83 \%$. Les taux d'analphabétisme les plus faibles avaient été observés à Bamako $(38 \%)$, Kayes $(58 \%)$, Kidal (69\%), Tombouctou (65\%) et Gao (72\%). L'enseignement coranique était prépondérant dans ses régions avec $22 \%$ à Tombouctou, $18 \%$ à Gao, $16 \%$ à Kidal et $10 \%$ à Mopti.

\section{Etat de santé des mères}

Dans l'échantillon étudié, $84 \%$ des femmes en âge de procréer étaient des mères dont $13 \%$ enceintes, $62 \%$ allaitaient et $25 \%$ n'étainet ni enceintes ni allaitant.Parmi les femmes enceintes, $41 \%$ étaient à leur troisième trimestre, $39 \%$ au second et $20 \%$ au premier trimestre de grossesse. Chaque femme de l'échantillon avait fait en moyenne 5 grossesses et 3 enfants vivants (Tableau 1). Bamako, Gao et Tombouctou avaient enregistré les plus faibles moyennes de grossesses et d'enfants vivants par femme. Un maximum de 20 grossesses et 13 enfants vivants par femme avaient été observés. Le nombre moyen d'enfant vivant par femme est resté toujours inférieur au nombre moyen de grossesses dans toutes les régions. Le nombre moyen d'enfant vivant par femme a évolué selon l'âge, mais est resté inférieur au nombre moyen de grossesse.

\section{Cécité crépusculaire et de l'anémie chez les Femmes} en âge de procréer

La prévalence de la cécité crépusculaire est estimée au niveau national à près de $6 \%$ avec un pic important à Tombouctou (14\%). Quant à l'anémie clinique, sa prévalence au niveau national est de $20 \%$ avec un niveau plus important à Koulikoro (40\%) et Mopti $(28 \%)$. Le taux le plus bas est enregistré à Kidal (4\%).

\section{Consultations et soins prénataux}

Près de $59 \%$ des femmes enceintes avaient fait au moins une consultation prénatale (CPN) munie de carnet au cours de leur grossesse (graphique 1). La possession du carnet de CPN était meilleure à Bamako avec 78,4\%, suivi de Koulikoro (77\%) et Kidal $(69,4 \%)$. Gao et Ségou avaient enrégistré les taux les plus faible avec respectivement $455 \%$ et $46,6 \%$. Parmi les $59 \%$ de femmes enceintes ayant fait des CPN, seules $34,8 \%$ avaient réalisé au moins $3 \mathrm{CPN}$ au cours de leur grossesse, $33,8 \%$ deux CPN et $31,5 \%$ une seule CPN. Seulement 3,5\% de femmes enceintes avaient fait leur 1 ère visite prénatale au cours du premier trimestre de la grossesse. Elles n'étaient que $24,6 \%$ à réaliser 3 visites prénatales au 3 ème trimestre de leur grossesse.

\section{Le Statut vaccinal des femmes enceintes}

Le taux de couverture vaccinale antitétanique des femmes enceintes au niveau national est de $67 \%$, pour le VAT 1 , et $51 \%$ pour le VAT 2 et plus. La vaccination antitétanique de rappel n'est pratiquée que par $33 \%$. 
Bamako (76\%), Gao (70\%), Kidal $(64 \%)$ et Koulikoro $(62 \%)$ ont respectivement les meilleurs taux de couverture en VAT2. Pour le rappel, Gao, Kidal et Bamako enregistrent respectivement les meilleurs taux.

\section{La Supplémentation en micronutriments}

Près de $80 \%$ des ménages maliens consomment du sel iodé. Seules les régions du Nord et de Kayes ont un niveau de supplémentation inférieur à la moyenne nationale $(49,9 \%)$. Gao enregistre le plus faible taux avec $11 \%$ / Ce sont les régions de Mopti (94\%), Sikasso (92\%) et le District de Bamako (91\%) qui affichent les meilleurs niveaux de consommation de sel iodé (tableau 2). $44 \%$ de mères avaient déclaré avoir bénéficié d'une supplémentation en vitamine $\mathrm{A}$ dans le post-partum. Bamako $(71,4 \%)$, Kidal $(50,4 \%)$, Ségou $(47,2 \%)$ et Sikasso $(46,2 \%)$ ont les meilleurs taux de supplémentation. Mopti $(20,6 \%)$ et Gao $(34,1 \%)$ affichent les plus faibles taux (Tableau 2).

Le niveau de supplémentation en fer des femmes enceintes est de 50\% (tableau 2) avec le plus faible taux enregistré à Tombouctou (32\%) et Ségou (36\%).

La prévention du paludisme pendant la grossesse : La Sulfadoxine pyriméthamine (SP) a été utilisée au niveau national dans la prévention du paludisme de la grossesse par $37 \%$ des femmes enceintes. Dans $23 \%$ des cas (tableau 3), un autre antipaludéen est utilisé comme moyen prophylactique. Environ $60 \%$ des femmes enceintes avaient utilisé un médicament comme moyen prophylactique. Le niveau d'utilisation de la SP, actuellement recommandée pour la prévention du paludisme de la femme enceinte n'tait pas satisfaisant $(37,4 \%)$. Dans toutes les régions du pays (tableau 3). II a été reste en dessous de $50 \%$ A Gao, son niveau d'utilisation est même nul. Un autre antipaludéen lui est préféré par plus de $60 \%$. Dans les régions de Ségou $(53,9 \%)$, Kayes $(48,6 \%)$ et Tombouctou $(47,5 \%)$, des proportions assez importantes de femmes enceintes n'utilisent encore aucun médicament pour la prévention du paludisme (tableau 3).

Statut nutritionnel des enfants :

L'étude avait porté sur 7259 enfants âgés de 0 à 59 mois. L'émaciation a concerné $10 \%$ des enfants de moins de 5 ans au niveau national dont $1 \%$ de sa forme sévère. Elle touche indifféremment les garçons (10,3\%) et les filles $(9,1 \%)$ avec $p>0,05$. Le retard de croissance a concerné $25 \%$ des enfants dont $8 \%$ font la forme sévère, frappant indifféremment les filles $(23,8 \%)$ et les garçons $(26,7 \%)$. L'insuffisance pondérale qui était de $26 \%$ dont de $8 \%$ de forme sévère touchait aussi bien les garçons $(27,2 \%)$ que les filles $(25,2 \%) p>0,05$.

Statut nutritionnel des enfants et niveau d'instruction de leurs mères :

L'émaciation frappait beaucoup plus les enfants issus de mères sans instruction $(11,9 \%)$ ou n'ayant atteint que le niveau primaire $(12,7 \%)$ ou simplement alphabétisées $(11,2 \%)$ contrairement à ceux dont les mères ont un niveau d'instruction assez élevé $(6,3 \%$ pour le niveau fondamental et $4,3 \%$ pour le supérieur) $p<0,001$.
La prévalence du retard de croissance diminue avec le niveau d'instruction des mères $(16,9 \%$ chez les enfants de mères ayant le niveau primaire, $8,2 \%$ chez ceux dont les mères ont un niveau secondaire) par contre il était très élevé chez les enfants de mères peu instruites $(33,8$ $\%$ pour les alphabétisées, $27,1 \%$ pour les non instruites et $22,6 \%$ pour le niveau coranique) $p<0,000$. L'insuffisance pondérale suit la même logique.

Le statut matrimonial de la mère agit sur l'état nutritionnel des enfants. Ainsi, les enfants nés de mères célibataires sont statistiquement plus touchés par l'émaciation $(15,4 \%$ chez les enfants nés de mères célibataires contre $9,7 \%$ chez les mariées) $p<0,000$. Le retard de croissance par contre frappe plus les enfants de mères mariées $(25,4 \%)$, veuves $(24 \%)$ ou divorcée ou en séparation de corps $(19,4 \%)$ contrairement aux enfants nés de mères célibataires $(13,1 \%) p<0,001$.

La proportion d'enfants émaciés augmente très rapidement en fonction de l'âge. De $3 \%$ pour les enfants de moins de 6 mois, la prévalence augmente pour atteindre son pic de $23 \%$ à $12-17$ mois (graphique 2). A partir de ce groupe d'âge elle diminue considérablement jusqu'à $15 \%$ à $18-23$ mois pour se retrouver à $5 \%$ entre 48-59 mois (graphique2).

Le retard de croissance touche plus deux enfants sur cinq soit $41 \%$ de la tranche d'âge $18-23$ mois et $29 \%$ de ceux de 12 à 17 mois (graphique 3 ).

La prévalence de l'émaciation selon les régions du pays indiquait un niveau plus élevé à Gao $(16,1 \%)$ et à Kayes $(14,3 \%)$. Par contre, les régions de Sikasso (7,8\%), Mopti $(7,8 \%)$ et le district de Bamako $(7,9 \%)$ étaient les moins touchées (graphique 4). Le retard de croissance par contre frappait plus les région de Sikasso $(33,4 \%)$, Ségou $(28,5 \%)$, Mopti $(25,8 \%)$ et Koulikoro $(28,1 \%)$ pendant que celles de Kidal (12,8\%), Bamako (13,8\%) et Gao étaient les moins touchées (graphique 5)

La prévalence de l'émaciation est significativement plus importante chez les enfants issus des ménages dirigés par une femme (tableau 4), contrairement aux deux autres types de malnutrition qui sont plus fréquents dans les ménages ayant à tête des hommes $(p=0,000)$.

Le statut nutritionnel des mères montrait que $8,3 \%$ d'entre elles présentaient un déficit énergétique chronique avec un niveau de prévalence plus marqué chez les plus jeunes avec $11,5 \%$ pour les moins de 19 ans et 10,4\% pour les 20 à 24 ans (tableau 5). L'obésité par contre, avec un niveau national de 5,9\% frappait plus les femmes plus âgées avec un pic chez les $35-39$ ans $(28,7 \%)$ suivi des $30-34$ ans $(13,3 \%)$ et les $45-49$ ans $(10,5 \%)$ (tableau5).

Le statut matrimonial de la femme influence sont état nutritionnel. Ainsi, les femmes célibataires étaient plus sujettes au déficit énergétique chronique (10,9\%) comparativement aux autres (tableau 6). La surcharge pondérale par contre s'observait plus chez les femmes divorcées ou séparées $(32,4 \%)$ contre $3 \%$ pour les célibataires et 5,9\% pour les mariées (tableau 6).

Selon les régions, il a été constaté que le déficit énergétique chronique frappait surtout les femmes de Gao (13,7\%), Tombouctou et Koulikoro (11,6\% chacune), 
contrairement aux régions de Kidal $(4,4 \%)$ et Sikasso (4,5\%)(Tableau 7). L'obésité par contre frappait plus les femmes de la région de Kidal $(24,1 \%)$, soit près d'une femme sur quatre et celles du district de Bamako $(1,9 \%)$ à l'opposé de celles de Sikasso $(0,9 \%)$ et Koulikoro $(1,6 \%)$ (Tableau 7). Selon le système de vie, les femmes qui vivaient dans les systèmes de vie riz de submersion et pasteur étaient les plus atteintes par le déficit énergétique chronique (14\% chacune) suivie de celles qui sont dans l'agro-migration $13 \%$, culture décrue et l'agro-rente (12\% chacune). L(obésité par contre se contre plus dans les milieu de vie pasteur $(15,7 \%)$ et agro-pasteur $(11,9 \%)$.

\section{DISCUSSIONS :}

Chez les enfants de moins de 5 ans :

Par rapport à l'état de santé des enfants, notre étude a noté une plus forte couverture vaccinale par rapport aux autres enquêtes nationales depuis de 2001. Selon EDS IV 2006 la proportion d'enfants vaccinés contre des maladies cibles du PEV a passé de $29 \%$ à $48 \%$ en 2001 contre $66 \%$ (2006) d'enfants qui avaient leur vaccination à jour dans notre étude [1]. Le taux de couverture vaccinale contre la rougeole s'élève à $69 \%$, ce taux est presque proche de celui observé par l'EDSM IV 2006 (68 $\%)$ [1].

La prévalence de la diarrhée est plus élevée que celle obtenue en 2005 par le CFSVA qui était de 11\% [2] et de l'EDSM IV 2006 de 19\% [2]. Comme lors du CFSVA avec respectivement $19 \%$ et $17 \%$, les enfants des classes d'âge de 12 à 23 mois et 6 à 11 mois sont les plus affectés par la diarrhée dans notre étude avec respectivement des taux de $28,7 \%$ et $25,8 \%$. La classe la moins affectée est celle des 48 - 59 mois avec $20 \%$ comme l'a observé l'enquête du CFSVA de 2005.

La prévalence des infections respiratoires (25\%) dans notre étude est nettement plus élevée que celle obtenue en 2005 par le CFSVA (7\%) [2].

Par rapport au statut nutritionnel des enfants:

On note une nette amélioration de l'allaitement exclusif des enfants de 0-6mois dans notre étude par rapport aux EDSM-III et IV-2006 qui donnent respectivement $25 \%$ et $38 \%$ contre $34 \%$ dans notre étude [3-1]. Le Mali se situe à un niveau plus élevé de proportions d'enfants 0-6 mois exclusivement allaités parmi les autres pays de l'Afrique subsaharienne. A l'opposé du Sénégal, où près de $6 \%$ seulement d'enfants de 0-6 mois étaient exclusivement allaités depuis 2006 [4], au Niger plus d'un enfant (0-6 mois) sur 6 étaient exclusivement allaités [5] et au Burkina Faso prés de $21 \%$ des enfants de moins de 6 mois étaient exclusivement allaité.

L'analyse comparée des données de la présente étude avec les autres études montre que nos résultats sont plus bas pour tous les types de malnutrition que ceux obtenus par les enquêtes démographiques et de santé de 2001 à 2006 et de celle du CFSVA 2005 :

Dans notre étude $10 \%$ des enfants de moins de 5 ans souffraient de malnutrition aigue ou l'émaciation contre $15 \%$ dans l'EDS -IV 2006 et $11 \%$ dans celle du CFSVA
[1-2]. $25 \%$ des enfants de moins de 5 ans souffraient de malnutrition chronique ou retard de croissance dans notre étude contre 38\% dans l'EDS-IV 2006 au Mali et $37,2 \%$ dans celle du CFSVA en 2005 [1-2].

$26 \%$ des enfants de moins de 5 ans souffraient de malnutrition globale ou insuffisance pondérale dans notre étude contre 27\% dans l'EDS-IV 2006 au Mali et 34,6\% dans CFCVA 2005 [1-2]. Parmi les autres pays d'Afrique subsaharienne ayant effectué la même étude le Mali est l'un des pays qui enregistre la plus faible proportion d'enfants émaciés; à l'opposé du Sénégal et de la Mauritanie où plus d'un enfant sur quatre soit $(29 \%)$ souffraient de l'émaciation depuis 2006 [4-6], du Niger $(10,3 \%)$ et du Burkina Faso (11\%) d'enfants émaciés en 2006 [5-7], la proportion d'enfant accusant de retard de croissance $(25 \%)$ dans notre étude est très proche de celle observée au Sénégal (27\%) [4] et au Burkina Faso (24\%), et inférieure à celle du Niger (50\%) [5-7] depuis 2006. Et enfin tous les autres pays de l'Afrique subsaharienne sauf le Sénégal ont un niveau de proportion d'enfants souffrants de l'insuffisance pondérale plus élevé que le Mali au Niger $(44,4 \%)$ au Burkina (29\%) et au Sénégal (21\%) [4-5-7]. La prévalence du retard de croissance est plus élevée chez les enfants de mères sans instructions dans l'EDSM-IV2006 tandis-que ce sont les mères alphabétisées qui ont plus d'enfants malnutris toutes formes confondues dans notre étude. Au Sénégal un enfant sur quatre dont la mère n'a reçue aucune instruction soit $(25 \%)$ est affecté par le retard de croissance [4]. Le niveau de l'insuffisance pondérale est presque trois fois plus élevé chez les enfants dont la mère n'a pas d'instruction que chez ceux dont la mère a un niveau d'instruction secondaire ou supérieur [4].

L'analyse de la situation nutritionnelle des enfants de 0 à 59 mois pendant cette période de soudure montre que :

- le taux de prévalence de la malnutrition aiguë $(10 \%)$ est jugé élevé comparé au seuil moyen de l'OMS (5 à $9 \%)$

- le taux de prévalence de la malnutrition chronique $(25 \%)$ est jugé moyen comparé au seuil moyen de l'OMS (20 à 29\%) [8],

- Et le taux de prévalence de l'insuffisance pondérale (26\%) est également jugé élevé comparé au seuil moyen de l'OMS (10 à 15\%) [8].

En somme, la situation nutritionnelle des enfants au Mali en cette période de soudure reste très critique aux regards des taux de prévalence des différents types de malnutrition comparés aux seuils de l'OMS [8].

\section{Chez les femmes en âge de procréer}

Les femmes de moins de 20 ans représentaient $14 \%$ dans notre étude contre $21 \%$ dans l'EDSM IV de 2006 et 9\% dans le CFSVA de 2005 [1-2].

Selon la région, dans notre étude, ce sont les régions de Koulikoro, Sikasso, Ségou et Mopti qui avaient enregistré respectivement les plus forts taux de femmes qui n'avaient reçue aucune instruction $(91 \%, 92 \%, 84 \%$, et $83 \%$ ) [1] par contre dans l'EDSM IV 2006 à l'exception de Bamako les proportions des femmes en âge de procréer sans instructions étaient partout élevées avec 
un minimum de $75 \%$ à Kidal à un maximum de $88 \%$ à Mopti.

Dans notre étude $94 \%$ des femmes étaient mariées et c'est le district de Bamako qui enregistre le plus fort taux de femmes célibataires avec $18 \%$ suivie de la région de Kidal avec $9 \%$.

Ces résultats concordent avec ceux observés par l'EDS IV 2006 au Mali et le CSFVA 2005 où les femmes mariées représentaient respectivement $93 \%$ et $92 \%$.

Etat de santé des mères :

La prévalence de la cécité crépusculaire $(6 \%)$ dans notre étude est la même que celle observée à dans EDSM-IV 2006 mais plus basse que celle obtenue au Sénégal $(16 \%)$ en 2006 [4]. Quant à l'anémie clinique, sa prévalence au niveau national est de $20 \%$ dans notre étude avec un niveau plus important à Koulikoro (40\%) et Mopti (28\%). Ce taux est inférieur à celui observée au Sénégal (23\%) [4], au Benin et au Niger avec 29\% chacune [5]. Le taux le plus bas est enregistré à Kidal (4\%) et cela concorde avec celui observé par une équipe de l'INSRP en 2006 (3,8\%) [9].

Par rapport à la supplémentation en micronutriment: Selon L'EDS IV 2006 au Mali il est apparu que dans l'ensemble $91 \%$ des ménages disposaient du sel iodé au Mali contre $(80 \%)$ des ménages qui consommaient du sel iodé dans notre étude. Dans l'EDSM IV [5], ce sont les régions de Kayes et Tombouctou que l'on observe la proportion la plus faible $(60 \%)$ chacune [1] tandis que toutes les régions du Nord et Kayes avaient un niveau de supplémentation inférieur à la moyenne nationale dans notre étude et Gao affichait le plus faible taux avec $11 \%$ seulement de mères qui utilisent le sel iodé dans leur alimentation. Le niveau de consommation très faible de sel iodé dans les régions du nord s'explique en grande partie par la consommation traditionnelle du sel gemme. Les régions de Mopti (94\%), Sikasso (92\%) et le District de Bamako (91\%) affichent les meilleurs taux dans les 2 études.

En ce qui concerne la prise en post partum de Vit A par les mères, dans I'EDS-IV 2006 au Mali, 41\% des mères ont répondues qu'elles avaient effectivement reçu de la Vit $A$ [1] contre $44 \%$ de mères qui ont déclaré d'avoir bénéficier d'une supplémentation en vitamine $A$ dans le post-partum dans notre étude. Dans notre étude ce sont les régions de Gao et Mopti qui affichaient les plus faibles taux de la prise de Vit A post partum alors que dans le CFSVA 2005 ce sont les régions de Kidal (18\%), Sikasso et Mopti (31\% chacune) qui enregistrent les plus faibles proportions des femmes qui ont reçues de Vit $A$ après leur accouchement. Les meilleurs taux de supplémentation ont été observé dans le district de Bamako $(67 \%)$ toutes les études confondues.

Le niveau de supplémentation en fer des femmes enceintes dans notre étude est de $50 \%$ dans notre étude contre $62 \%$ des femmes qui avaient déclaré l'avoir bénéficier dans l'EDSM IV 2006 [1] dont la plus forte proportion a été observée à Bamako $(28 \%)$ tandis-que à l'exception de Tombouctou (32\%) et de Ségou (36\%) toutes les régions avaient un niveau de supplémentation en fer supérieur ou égale à la moyenne $(50 \%)$ dans notre étude.

\section{Par rapport au statut nutritionnel :}

La prévalence du déficit énergétique chronique $(8 \%)$ est nettement plus basse que celle observée en 2006 par I'EDSM -IV (14\%) et au Niger (14\%) [5] mais très proche de celle retrouvée en 2005 au Sénégal (9\%) et au Bénin (7\%) [4-7]

La proportion de femmes obèses est légèrement plus importante dans notre étude $(6 \%)$ comparativement à l'enquête démographique et de santé de 2006 (EDSM IV) qui était de $5 \%$ [1]. Comme observé dans I'EDSM IV2006, les femmes jeunes sont plus malnutries que celles plus âgées (12\% chez les moins de 19 ans dans notre étude contre $22 \%$ en 2006 pour la même tranche d'âge) [1]. De même l'obésité frappe préférentiellement les femmes plus âgées dans toutes les études nationales confondues et on constate également que les femmes des régions de Gao $(14,2 \%)$, Kayes $(11,9 \%)$, Koulikoro $(11,23 \%)$ sont plus maigres que celles des autres régions. Quant à l'obésité, c'est la région de Kidal, le district de Bamako et la région de Tombouctou qui sont les plus touchées avec respectivement $(23,2 \%),(21,4 \%$ et $20,7 \%$ ).

\section{Conclusion et recommandations}

Les résultats de cette étude ont mis en évidence les déterminants de la malnutrition tels que les enfants des mères plus âgées et ceux des mères qui n'ont reçues aucunes instructions, sont les plus touchés par les différents types malnutrition; les différentes maladies de l'enfant au cours des sept derniers jours influent négativement son statut nutritionnel quelque soit le type ; plus les enfants sont moins couverts par la vaccination plus ils courent le risque d'être malnutris. L'analyse de la situation nutritionnelle des enfants montre des niveaux de prévalence élevés avec une forte disparité régionale. L'émaciation touche $9,7 \%$ des enfants de 0 à 5 ans avec $1 \%$ de forme sévère. Gao avec $16 \%$ est la région la plus affectée, suivie de celle de Kayes (14\%) tandis que Mopti est la région la moins affectée. Quant au retard de croissance, il touche $25 \%$ des enfants avec 8 $\%$ de forme sévère. La région de Kidal avec $13 \%$ et Bamako $14 \%$ sont les moins touchées. La région de Sikasso avec $33 \%$ est la plus affectée. L'insuffisance pondérale atteint $26 \%$ des enfants de 0 à 5 ans sur l'ensemble du territoire national. Cette proportion est plus faible dans la région de Kidal $(15 \%)$ et le district de Bamako (19\%). Elle est plus élevée dans les régions de Tombouctou (33\%) et Ségou (29\%). Quant aux femmes en âge de procréer, $8 \%$ d'entre présentent un déficit énergétique chronique contre $5,9 \%$ d'obèses. Cette situation nécessite le renforcement de la lutte contre les maladies infantiles à travers une meilleure couverture vaccinale, un meilleur accès à l'eau potable, le déparasitage systématique des enfants et la promotion de bonnes pratiques d'hygiène et de l'allaitement exclusif. 
Références bibliographiques:

1. Mali. Enquête Démographique et de Santé 2006, MS DNSI, Macro orc, juin 2007,

2. PAM/UNICEF. Analyse Globale de la Sécurité Alimentaire et de la Vulnérabilité de Malnutrition chez les Enfants de moins de 5 ans au Mali (2006).

3. CPSISANTE, DNSI. Enquête Démographique et de Santé, Juin 2002.

4. Ministère de la Santé Publique au Sénégal, DNSI, EDSS-IV 2006, Statut nutritionnel des enfants de moins de 5 ans et les carences en micronutriments des femmes à l'âge de procréer, 2006, 98p.

5. USAID. Profiles Nutrition au Niger, Etat nutritionnel des enfants de moins de 5 ans et les carences en micronutriments des femmes à l'âge de procréer, Niamey du 19 au 27 juin 2006, 7p.

6. PAM. Rapport sur le Suivi de la Situation Alimentaire en Mauritanie, février 2006.16p.

7. PAM. Evaluation de la sécurité alimentaire et nutritionnelle des populations rurales de la zone soudano-sahélienne au Benin et au Burkina Faso, Statut nutritionnel des enfants de moins de 5 ans carences en micronutriments des femmes à l'âge de procréer, juin 2006, 39p.
8. WHO, working group. Use and interpretation of anthropometric indicator of nutrition status. Bull Who 1995. 854:1-452.

9. MS, INRSP. Etude sur la rétinolemie à Bamako et à Koulikoro: Carence en Micronutriment chez les enfants d'âge scolaire et les femmes en âge de procréer, Institut National de la Recherche en Sante Publique, octobre 2001, 59p.

10. AG IKNANE A, BEN ALWATA C, DIARRA S, et al. Enquête de base sur la sécurité alimentaire et la nutrition, Système d'Alerte Précoce, juin 2007, 95p.

11. Yacouba HA, Niandou H. Evaluation rapide de la situation nutritionnelle des enfants de 0-5 ans dans les régions de diffa, Zinder et Maradi frontalières du Nigéria, état nutritionnel des enfants des mères qui n'ont reçues aucunes instructions au Niger, 2007, $19 p$.

12. Pelletier DL, Frongillo EAJ, Schroeder DG, Habicht JP. "The effects of malnutrition on child mortality in developing countries." Bull World Health Organ. 1995.73p.

Tableau 4 : Répartition des différentes formes de malnutrition selon le chef de ménage

\begin{tabular}{|l|c|c|}
\hline \multirow{2}{*}{ Types de malnutrition } & \multicolumn{2}{|c|}{ Sexe du chef de ménage } \\
\cline { 2 - 3 } & Masculin (\%) & Féminin (\%) \\
\hline Emaciation & 9,7 & 12,4 \\
\hline Retard de croissance & 25,7 & 18,3 \\
\hline Insuffisance Pondérale & 26,8 & 21,8 \\
\hline
\end{tabular}


Tableau 1: Répartition du nombre moyen de grossesse et d'enfants vivants par femme selon la tranche d'âge

\begin{tabular}{|l|c|c|}
\hline $\begin{array}{c}\text { Classe } \\
\text { d'âge }\end{array}$ & $\begin{array}{c}\text { Nombre } \\
\text { moyen de } \\
\text { grossesse }\end{array}$ & $\begin{array}{c}\text { Nombre } \\
\text { moyen } \\
\text { d'enfants } \\
\text { vivants }\end{array}$ \\
\hline$<=19$ & 1,3 & 1,1 \\
\hline $20-24$ & 2,5 & 2 \\
\hline $25-29$ & 3,8 & 3 \\
\hline $30-34$ & 5,3 & 4,1 \\
\hline $35-39$ & 6,5 & 5 \\
\hline $40-44$ & 7,4 & 5,5 \\
\hline $45-49$ & 8,4 & 5,8 \\
\hline Moyenne & 4,3 & 3,3 \\
\hline
\end{tabular}

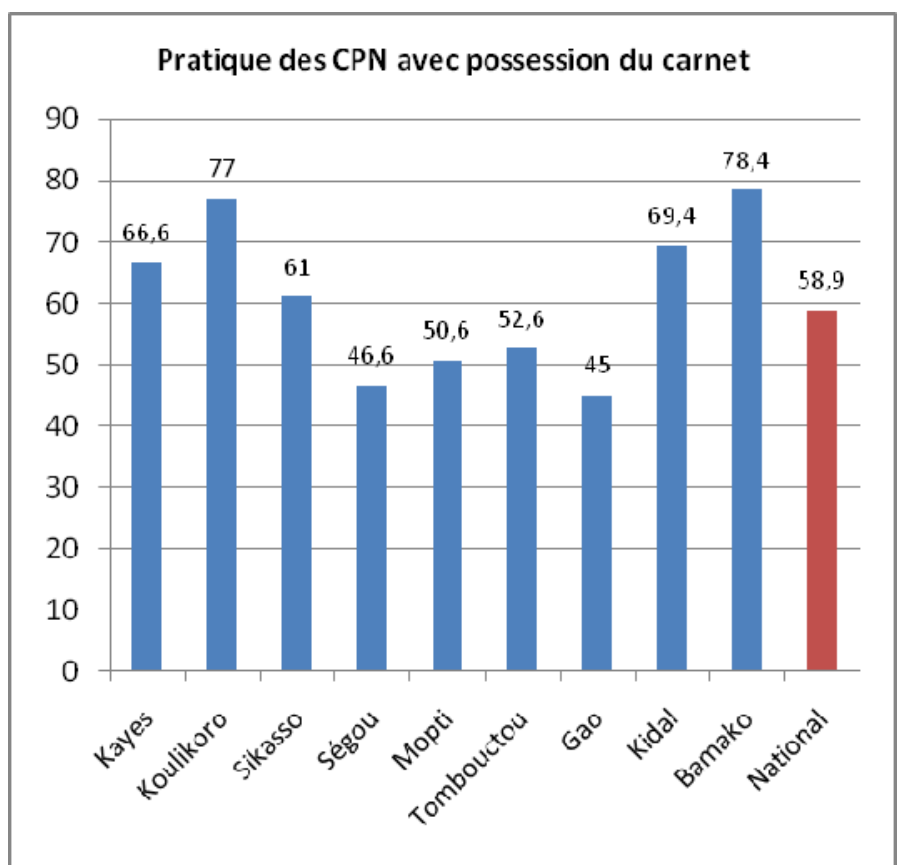

Graphique 1 : Pratique de la CPN avec carnet selon leur provenance
Tableau 2 : Répartition de la supplémentation en micronutriments des mères selon les régions

\begin{tabular}{|l|c|c|c|}
\hline Régions & $\begin{array}{c}\text { Consommation } \\
\text { sel iodé }\end{array}$ & $\begin{array}{c}\text { Vit A en post- } \\
\text { partumt }\end{array}$ & $\begin{array}{c}\text { Fer pendant } \\
\text { la } \\
\text { grossesse }\end{array}$ \\
\hline Kayes & 59,5 & 40,7 & 49,4 \\
\hline Koulikoro & 70,1 & 37,5 & 56,6 \\
\hline Sikasso & 92,1 & 46,2 & 59,2 \\
\hline Ségou & 88,9 & 47,2 & 35,6 \\
\hline Mopti & 94,1 & 20,6 & 43,5 \\
\hline Tombouctou & 49,9 & 39,8 & 32,0 \\
\hline Gao & 10,8 & 34,1 & 67,9 \\
\hline Kidal & $\mathbf{5 1 , 0}$ & $\mathbf{5 0 , 4}$ & $\mathbf{5 9 , 6}$ \\
\hline Bamako & 90,6 & 71,4 & 59,9 \\
\hline Moyenne & 79.7 & 43,9 & 49,9 \\
\hline
\end{tabular}

Tableau 3 : Répartition de la prise de médicaments pour la prévention du paludisme par des femmes enceintes selon la région

\begin{tabular}{|l|c|c|c|}
\hline REGIONS & $\begin{array}{c}\text { SP (Sulfadoxine } \\
- \\
\text { Pyriméthamine) }\end{array}$ & $\begin{array}{c}\text { Autre } \\
\text { médicament }\end{array}$ & Rien \\
\hline Kayes & 43,6 & 7,8 & $\mathbf{4 8 , 6}$ \\
\hline Koulikoro & 47,7 & 11,3 & 41,0 \\
\hline Sikasso & 31,7 & 33,7 & 34,7 \\
\hline Ségou & 32,2 & 13,9 & $\mathbf{5 3 , 9}$ \\
\hline Mopti & 37,8 & 25,8 & 36,4 \\
\hline Tombouctou & 29,2 & 23,4 & $\mathbf{4 7 , 5}$ \\
\hline Gao & 0 & $\mathbf{6 8 , 9}$ & 31,1 \\
\hline Kidal & 33,3 & 29,9 & 36,9 \\
\hline Bamako & 38,2 & 43,3 & 18,5 \\
\hline Moyenne & $\mathbf{3 7 , 4}$ & $\mathbf{2 2 , 9}$ & $\mathbf{3 9 , 7}$ \\
\hline
\end{tabular}


Tableau 7 : Prévalence du déficit énergétique chronique et de l'obésité chez les mères selon la région

\begin{tabular}{|l|c|c|c|c|}
\hline Régions : & $\begin{array}{l}\text { DEC : } \\
I M C<18,5\end{array}$ & $\begin{array}{l}\text { Risque de } \\
\text { DEC : } \\
I M C>=18,5 \\
\text { et }<20\end{array}$ & $\begin{array}{l}\text { Risque } \\
\text { d'obésité : } \\
I M C>25 \\
\text { et }>30\end{array}$ & $\begin{array}{l}\text { Obésité : } \\
I M C>30\end{array}$ \\
\hline Kayes & $\mathbf{1 0 , 8}$ & $\mathbf{1 9 , 1}$ & 12,4 & 4,2 \\
\hline Koulikoro & $\mathbf{1 1 , 6}$ & $\mathbf{1 8}$ & 11,4 & 1,6 \\
\hline Sikasso & 4,5 & 12,1 & 8,2 & 0,9 \\
\hline Ségou & 8,6 & 13,4 & 13,1 & 3,3 \\
\hline Mopti & $\mathbf{1 0 , 8}$ & 12,3 & 13,8 & 4,3 \\
\hline Tombouctou & $\mathbf{1 1 , 6}$ & 12,4 & $\mathbf{2 3 , 9}$ & 13,9 \\
\hline Gao & $\mathbf{1 3 , 7}$ & 10,5 & $\mathbf{2 3 , 6}$ & 13,3 \\
\hline Kidal & 4,4 & $\mathbf{7 , 6}$ & $\mathbf{2 8 , 7}$ & $\mathbf{2 4 , 1}$ \\
\hline Bamako & $\mathbf{8 , 8}$ & 10,1 & $\mathbf{1 8 , 8}$ & $\mathbf{1 7 , 9}$ \\
\hline Moyenne & $\mathbf{8 , 3}$ & $\mathbf{1 4 , 1}$ & $\mathbf{1 3 , 7}$ & $\mathbf{5 , 9}$ \\
\hline
\end{tabular}

Tableau 8: Prévalence du déficit énergétique chronique et de l'obésité chez les mères selon le système de vie

\begin{tabular}{|l|c|c|c|c|}
\hline Systèmes de vies & $\begin{array}{c}\text { DEC : } \\
\text { IMC< } \\
\mathbf{1 8 , 5}\end{array}$ & $\begin{array}{c}\text { Risque } \\
\text { de DEC : } \\
\text { IMC } \\
\mathbf{= 1 8 , 5} \text { et } \\
<\mathbf{2 0}\end{array}$ & $\begin{array}{c}\text { Risque } \\
\text { d'obésité : } \\
\text { IMC entre } \\
\mathbf{2 5} \text { et } \mathbf{3 0}\end{array}$ & $\begin{array}{c}\text { Obésité : } \\
\text { IMC }>\mathbf{3 0}\end{array}$ \\
\hline Agro-coton & 8,7 & 15,2 & 8,3 & 1,5 \\
\hline Agro-coton_fruit & 6,7 & 14,2 & 7,8 & 1,2 \\
\hline Agro-élevage & $\mathbf{7 , 9}$ & 15,2 & 9,6 & 1,4 \\
\hline Agro-migration & $\mathbf{1 2 , 5}$ & $\mathbf{1 9 , 5}$ & 11,3 & 2,9 \\
\hline Agro-oignon & $\mathbf{4 , 9}$ & 11 & 12,2 & 1,8 \\
\hline Agro-pasteur & $\mathbf{1 0 , 9}$ & $\mathbf{7 , 3}$ & 18,4 & $\mathbf{1 1 , 9}$ \\
\hline Agro-rente & $\mathbf{1 1 , 5}$ & $\mathbf{1 8 , 8}$ & 8,4 & 0,9 \\
\hline Culture décrue & $\mathbf{1 1 , 6}$ & $\mathbf{1 6 , 6}$ & 17,5 & 4,3 \\
\hline Pasteur & $\mathbf{1 4 , 1}$ & $\mathbf{1 7 , 6}$ & 12,7 & $\mathbf{1 5 , 7}$ \\
\hline Riz Irrigué (ON) & $\mathbf{5 , 3}$ & 11,7 & 18,1 & 5,8 \\
\hline Riz de submersion & $\mathbf{1 4 , 2}$ & 13,8 & 18,6 & 4,5 \\
\hline Salariat/commerce & $\mathbf{7 , 2}$ & 9,9 & 20 & 14,3 \\
\hline Moyenne & $\mathbf{9 . 1}$ & 15.1 & 13.6 & 6.1 \\
\hline
\end{tabular}

